\title{
Clusters Merging Method for Short Texts Clustering
}

\author{
Yu Wang, Lihui Wu, Hongyu Shao \\ School of Management Science and Engineering, Dalian University of Technology, Dalian, China \\ Email: ywang@dlut.edu.cn, 876520946@qq.com, hong-yu-8@163.com
}

Received April 2014

\begin{abstract}
Under push of Mobile Internet, new social media such as microblog, we chat, question answering systems are constantly emerging. They produce huge amounts of short texts which bring forward new challenges to text clustering. In response to the features of large amount and dynamic growth of short texts, a two-stage clustering method was putted forward. This method adopted a sliding window sliding on the flow of short texts. Inside the slide window, hierarchical clustering method was used, and between the slide windows, clusters merging method based on information gain was adopted. Experiment indicated that this method is fast and has a higher accuracy.
\end{abstract}

\section{Keywords}

Short Texts Clustering, Slide Window, Information Gain, Hierarchical Clustering

\section{Introduction}

Mobile Internet era has arrived with the widespread use of mobile devices, especially smartphones. The increasing amount of data generated by mobile Internet is bringing new challenges and opportunities to data analysis, especially to data mining. Social medias based on mobile Internet like we chat, micro-blog, twitter and other applications have become increasingly popular, which produce a large number of information every day. The key here is that most of the information is appeared in the form of short text. In addition, user-interactive question answering system has attracted more and more attentions, with the rapid development of Web2.0. However, in these interactive systems, there are a large number of similar or duplicate information, which are generally short and colloquial. The wide range emergence of short texts on the Internet has proposed new requirements to existing text clustering methods. By cluster analysis, we can get hot news, organize short texts effectively, and build automated question answering systems.

Different from traditional text clustering, short text clustering is usually of the following characteristics [1]:

(1) Grow dynamically. Wechat, microblog and other emerging social medias have a rapid growth rate, but also have high real-time requirements on clustering results.

(2) Text is short. Usually there are fewer words in short texts, for example, Sina Weibo requires each message is no more than 140 words. This feature makes short texts fewer text features.

(3) Language is more casual, and there are many spoken language, abbreviations, new words, even spelling errors. 
(4) The number of short texts is huge, usually at least one million.

Traditional text clustering methods are mainly segmentation methods and hierarchical clustering methods.

The main idea of segmentation methods is to optimize existing clusters through different subdivision strategies. K-means algorithm is a typical segmentation algorithm [2], and it selects initial point as cluster center to continually iterate. K-means algorithm is easy to understand, easy to implement and is of a high efficiency. However, before starting to cluster, we must first specify the number of clusters, which is difficult to implement for large-scale dynamic growth short text data.

Hierarchical clustering algorithm [3] can be divided into hierarchical clustering and divisive hierarchical clustering based on clustering direction. It does not need to specify the number of clusters, but its time complexity is $\mathrm{O}\left(\mathrm{n}^{3}\right)$. So it cannot be applied to large-scale short text clustering.

For the text feature representation, traditional methods often use vector space model (VSM). The methods cannot extract all the features for short text expression. In addition, because terms in short texts are not standard, the results of segmentation are unsatisfactory. On this basis, Zhao Peng et al. [4] applied HowNet on the text semantic representation, so that the documents with the same theme can be merged.

In recent years, clustering algorithms for short texts are constantly raised. Jiliang Tang et al. [5] proposed a multi-language representation method for short texts using machine translation, extended text representation, and solved some problems like polysemes, synonyms and insufficient statistical information. Wang et al. [6] proposed an extension of vector space model for communication short texts, called WR-KMeans. Pengze Ying [7] found a short text clustering phenomenon by analyzing results of short texts clustering, which is called long-tail phenomenon. They applied long-tail phenomenon on clustering, removed some unnecessary clustering operations, and proposed an incomplete clustering method. Chen Jianchao [8] proposed an improved CBC algorithm, which adaptively determined the center of each cluster in its totality. All the methods are mainly focused on how to carry out feature representation and similarity calculation of short texts, while the clustering performance is not much improved.

This paper proposed a two-stage clustering method for dynamically increasing short texts. By windows sliding on the text streams, data inside the window performed hierarchical clustering, then similar clusters were merged on the results of hierarchical clustering between windows.

\section{Two-Stage Clustering Method}

For large-scale short texts, this paper decomposed them for enhancing cluster efficiently. Figure 1 shows the process of clustering.

In this method, a sliding window slides successively in those data which have not been clustered. It controls the number of objects to be clustered by setting the size of sliding window. It is hard to determine the number of clusters before clustering, so we choose agglomeration hierarchical clustering method. The drawback of hierar-

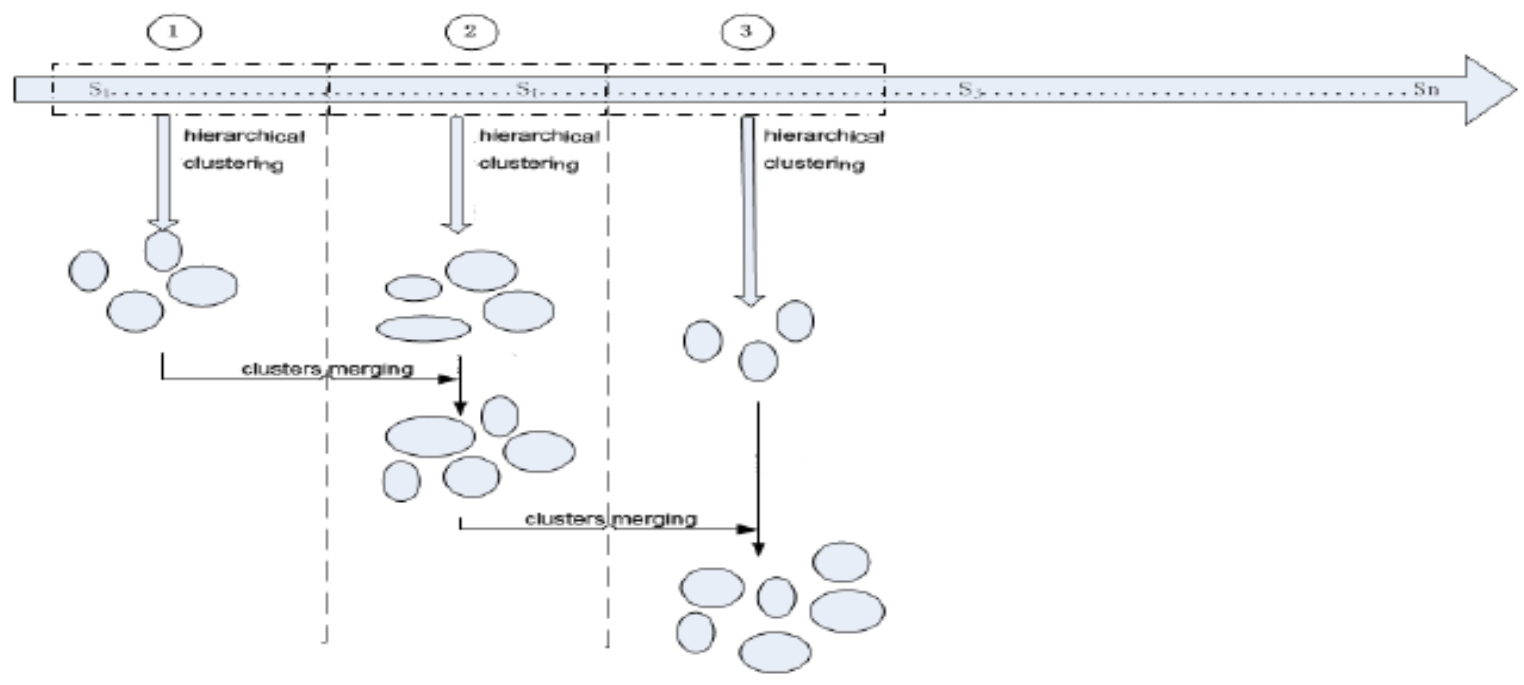

Figure 1. Two-stage clustering process. 
chical clustering method in time complexity can be overcome by setting a smaller size of sliding window. Classical vector space model is applied in short text representation. In order to improve the accuracy of segmentation, we need to add user dictionary and network words to segmentation dictionary in the process.

In the two-stage clustering, the first stage is hierarchical clustering, and then merge the clusters obtained by hierarchical clustering by cluster merging algorithm. This method improved the efficiency of large-scale text clustering. On the other hand, it can cluster increasing data in real time without the need of re-clustering the whole data sets.

\section{Cluster Merging Algorithm}

Previous studies rarely concerned cluster merging method. Hierarchical clustering calculated the similarity between two clusters by single-link, full-links or average-link. Liu Zhangxiong [9] divided data on a grid, and merged clusters on the basis of grid characteristics. These methods are essentially based on distance, but they measure distance only by a single point or representative point when comparing clusters, without considering the overall information of clusters, which will lead to two dissimilar clusters merging. Keeping iterating like this will ultimately reduce the accuracy of cluster merging.

We reconsidered cluster merging algorithm from the perspective of information theory, mainly based on the following basic knowledge: if two clusters are of high similarity, after one cluster merging into the other one, the information entropy of the latter will not increase by a large margin.

Corollary 1: Let B and C are two clusters to merge, and $\alpha$ is a specific constant. If $H(B \cup C)-H(B) \leq \alpha$, $\mathrm{B}$ and $\mathrm{C}$ are of a high similarity, and they can be merged. If $H(B \cup C)-H(B) \geq \alpha, \mathrm{B}$ and $\mathrm{C}$ are of a low similarity, and they cannot be merged.

\subsection{Calculating Information Entropy of Clusters}

In ID3 algorithm [10], there are decision attributes in training data sets, which can be used as classification label to get the information entropy. The paper extracted important words from each cluster as classification attributes to calculate the information entropy of each cluster.

Symbolic description: $B=\left\{S_{1}, S_{2}, \ldots, S_{m}\right\}$, a cluster sets contained $m$ short texts; $W B=\left\{W_{1}, W_{2}, \ldots, W_{p}\right\}$, result sets of short text segmentation of cluster $\mathrm{B}$; In $W_{i}=\left|\operatorname{Word}_{i}\right| / m \times i d f_{\text {wordi }}$, $\left|\operatorname{Word}_{\mathrm{i}}\right|$ is the number of sentences with "'Word" in cluster $B$, and idf $_{\text {wordi }}$ can be calculated on corpus; $C=\left\{S_{1}, S_{2}, \ldots, S_{n}\right\}$, a short text cluster set to merge; $W C=\left\{W_{1}, W_{2}, \ldots, W_{q}\right\}$ is similar with $W B$.

\section{Information Entropy Algorithm of Clusters $\mathrm{H}(B)$ :}

Input: $\mathrm{B}, \mathrm{WB}$ and mutual information threshold $\alpha$

Output: $\mathrm{H}(\mathrm{B})$

Begin

(1) Choose the two maximum values of Wi in WB as word1 and word $_{2}$.

(2) Calculate the mutual information of word 1 and word 2 in short text sets, and proceed 0 - 1 standardization for them, as Formula (1).

$$
\operatorname{MI}\left(\operatorname{Word}_{1}, \operatorname{Word}_{2}\right)=\log _{2} \frac{A \times N}{B \times C} / \log _{2} N
$$

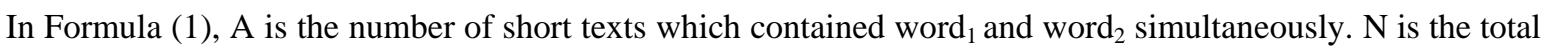
number of sentences in short texts. B is the number of short texts contained word ${ }_{1}$, and $C$ is the number of short texts contained word 2 .

(3) Divide equivalence classes on B by property $\mathrm{P}$ as $\mathrm{B} / \mathrm{P}=$ \{Join, First, Second, Noappear $\}$. Join represents

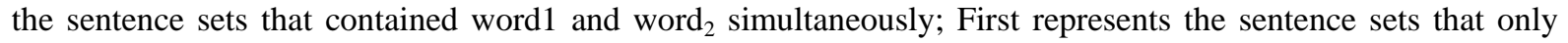
contained word1 and second represents the sentence sets that only contained word $_{2}$. Noappear is the sentence sets contained none of them.

(4) Merge Join, First and Second if MI(Word1, $\left.\operatorname{Word}_{2}\right)>\alpha$, that is $\mathrm{B} / \mathrm{P}=\{$ marge, noappear $\}$. Otherwise, keep unchanged.

(5) Calculate the information entropy of B as Formula (2).

$$
H(B)=-\sum_{c \in B / P} \frac{|c|}{m} \times \log _{2} \frac{|c|}{m}
$$


End

\subsection{Algorithm Design}

The above algorithm calculated the correlation between two words by mutual information, and then decided whether to merge the two words to divide equivalence classes. This kind of algorithmic idea can be also applied to calculating the information entropy of merging result of two clusters. When a cluster contained few short texts merge into a cluster contained more short texts, regardless of whether they are similar, the information entropy will not change a lot. Therefore, we only need to enlarge one cluster in proportion when calculating information entropy of merging results of two clusters. Cluster merging algorithm is as Figure 2.

\subsection{Algorithm Analysis}

Let the total number of short texts is $\mathrm{N}$, and set the size of window is $\mathrm{m}$. So the number of times of hierarchical clustering is $\left\lceil\frac{N}{m}\right\rceil$, and each time the results of hierarchical clustering need to be merged into the base clusters formed previously. First, carry out hierarchical clustering on short texts in the window, and the time complexity is $\mathrm{O}\left(\mathrm{m}^{3}\right)$. Set hierarchical clustering forms k clusters, and all the k clusters need to be merged into the base clusters formed previously in turn. The number of short texts in the base class sets will increase with the number of times of merging. Complexity of the $i$-th merging is $k^{*} i^{*} m$. Therefore, time complexity of every hierarchical clustering and merging is $\mathrm{O}\left(\mathrm{m}^{3}\right)+k^{*} i * m$, then there will be the formula (3) after $\mathrm{N} / \mathrm{m}$ times iteration:

$$
\left\lceil\frac{N}{m}\right\rceil \times O\left(m^{3}\right)+\sum_{i=1}^{\left\lceil\frac{N}{m}\right\rceil} k \times i \times m
$$

In order to express easily, ignore the subtle influence of rounding. Adjusting formula (3), we can get the formula $O\left(\frac{k+2 m^{2}}{2} \times N+\frac{k}{2 m} \times N^{2}\right)$. Regarding $\mathrm{k}$ and $\mathrm{m}$ as constant, the ultimate time complexity is $\mathrm{O}\left(N^{2}\right)$, and the same is the case with CURE clustering algorithm. In particular, the size of the sliding window is at tens of thousands level, so the constant coefficient $\frac{k}{2 m}$ of $N^{2}$ is much smaller than 1 .

\section{Experiment and Analysis}

\subsection{Experiment Setups}

All the experiments were set up on a server, who is of Intel Xeon CPU E5-2620@2.00GHz, 8 cores, 13G mem-

\begin{tabular}{|c|c|}
\hline 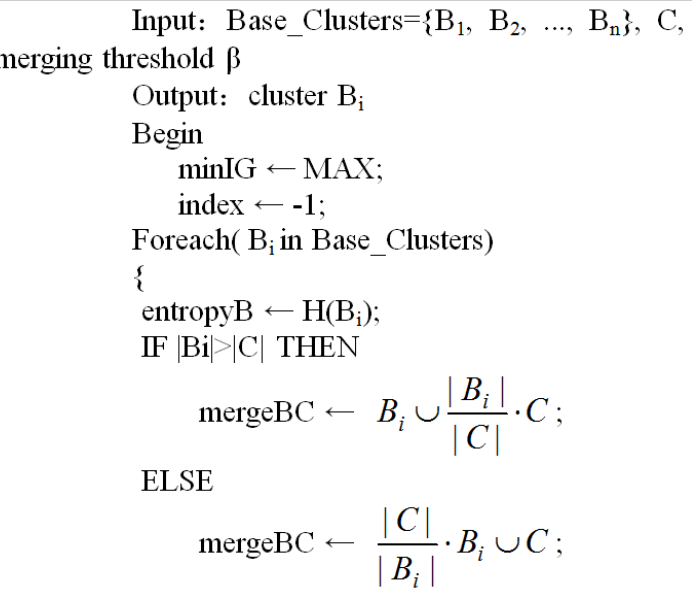 & 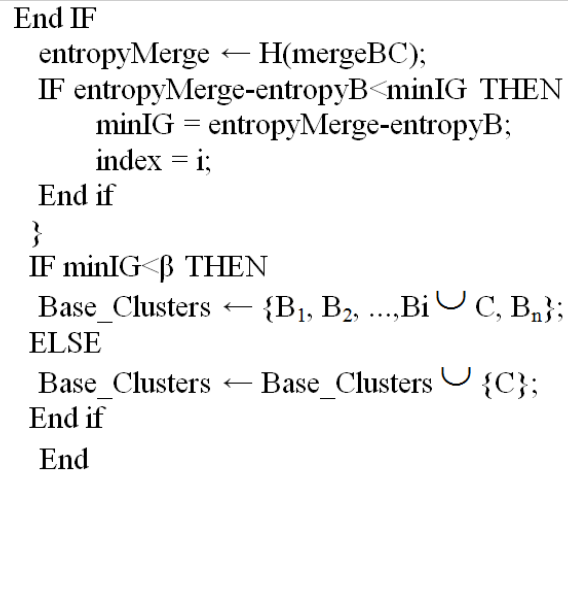 \\
\hline
\end{tabular}

Figure 2. Cluster Merging Algorithm. 
ory and Linux operating system. Algorithms were written in Java and programs were single-threaded. In order to prove the effectiveness and efficiency of our algorithms, we took the traditional CURE algorithm [11] as a reference.

All the data in this paper come from an infant education company, containing a total of 103,048 short texts on the issue of babies. 10,000 of them have been classified by experts and the infant education company. There are two interrelated evaluation criteria when analyzing clustering results: (1) the closer in a cluster the better, the more discrete between clusters the better; (2) the closer between clustering results and manual estimations the better [7]. In this paper, we used accuracy and standard mutual information two evaluation criterions to assess clustering results [12].

Let $l\left(c_{\mathrm{i}}\right)$ as the cluster label of cluster $\mathrm{c}_{\mathrm{i}}, l\left(\mathrm{~d}_{\mathrm{j}}\right)$ as the manual marking category of the $\mathrm{j}$-th short text, and the formula to definite the accuracy is as follows.

$$
A C C=\frac{1}{n} \sum_{i=1}^{k} \sum_{j=1}^{n} \delta\left(l\left(c_{i}\right), l\left(d_{j}\right)\right)
$$

and $\delta(x, y)=\left\{\begin{array}{ll}0 & x \neq y \\ 1 & x=y\end{array}\right.$.

Set C and C' are clusters, and definite mutual information MI(C,C') as Formula (5).

$$
\operatorname{MI}\left(C, C^{\prime}\right)=\sum_{c_{i} \in C, c^{\prime} \in C^{\prime}} p\left(c_{i}, c^{\prime}{ }_{j}\right) \log _{2} \frac{p\left(c_{i}, c^{\prime}{ }_{j}\right)}{p\left(c_{i}\right) p\left(c^{\prime}{ }_{j}\right)}
$$

Standard mutual information is as Formula (6)

$$
N M I\left(C, C^{\prime}\right)=\frac{M I\left(C, C^{\prime}\right)}{\max \left(H(C), H\left(C^{\prime}\right)\right)}
$$

Experiment analysis can be divided into three parts. First, set different mutual information thresholds and merging thresholds, examine the accuracy and changes on standard mutual information, and determine the optimal mutual information threshold and merging threshold. Second, compare clustering results and time complexity with CURE algorithm.

\subsection{Parameter Determination}

In cluster merging algorithm, it is also necessary to determine two parameters, mutual information threshold $\alpha$ and cluster merging threshold. When measuring $\alpha$ separately, set $\beta$ as 0.4 by estimating samples, then tested $\alpha$ from 0.1 to 0.9 to determine $\alpha$. After that, the parameters $\beta$ was determined. But it was so onerous to analyze 10000 short texts that we extracted 2500 data from them, and the results are shown in Figure 3 and Figure 4.

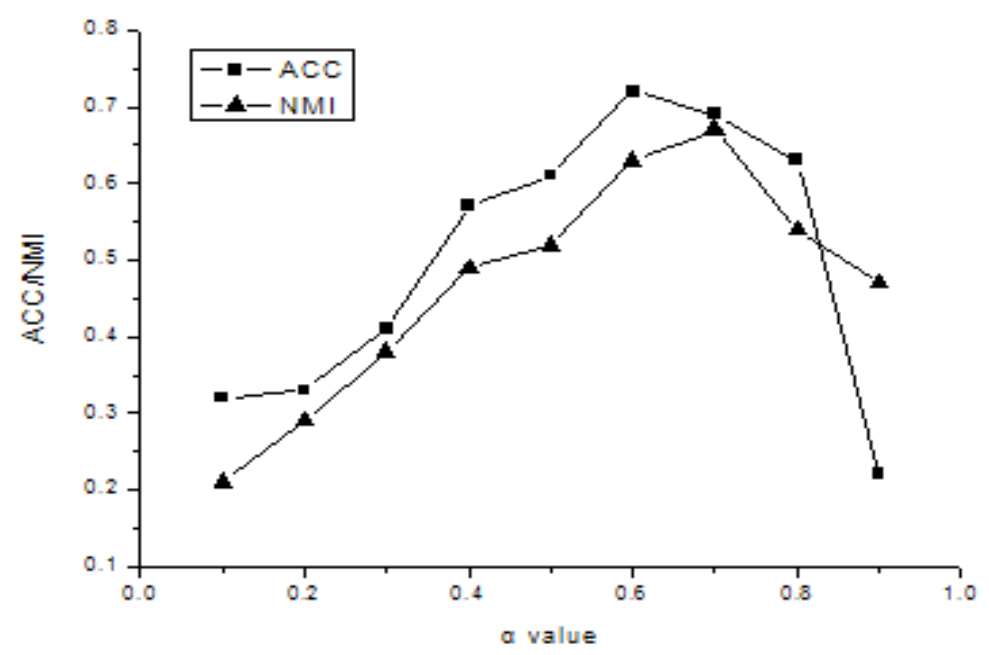

Figure 3. The ACC \& NMI under the changing $\alpha$. 
In Figure 3 and Figure 4, standard mutual information and accuracy have similar change trend. They increase first, then run down after reaching the peak. When the mutual information threshold value $\alpha$ is small, the possibility that mutual information of two words selected from clusters is larger than $\alpha$ increase, which reduce the number of categories obtained by dividing the short texts taking the two words as attributes, and the entropy calculated is generally small, and then unsimilar clusters will merge, forming some big cluster sets. Similarly, the value of $\beta$ will also lead to occurrence of the above situations. According to experiments, we finally determined $\alpha=0.7$ and $\beta=0.5$.

\subsection{Clustering Effect Analysis}

For the 10000 data, we marked artificially 133 categories, including 7238 short texts. The other 2762 sentences were isolated points or indeterminate, which were not included in the categories. Now the cluster in which the

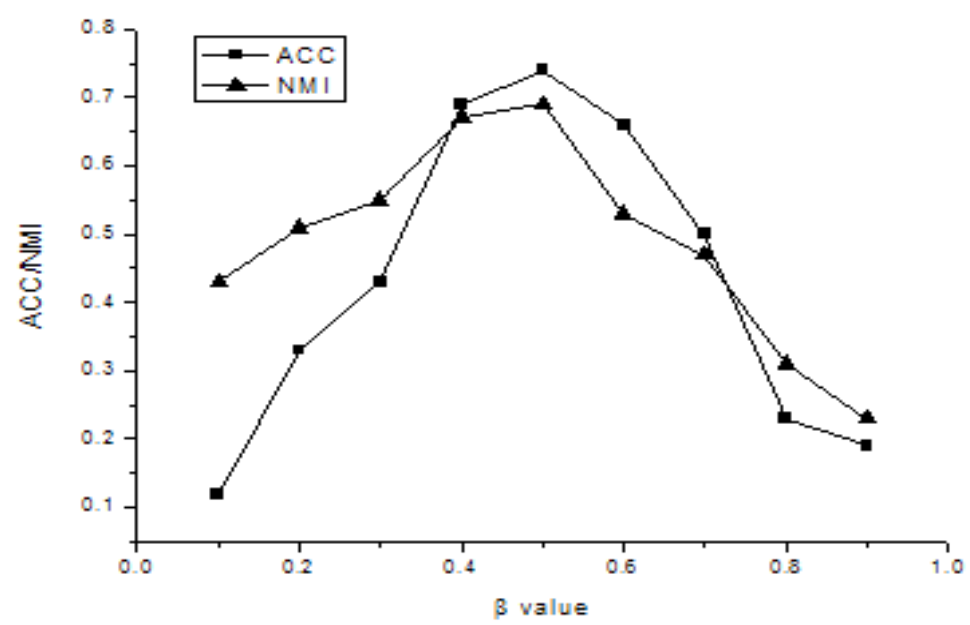

Figure 4. The ACC \& NMI under the changing $\beta$.

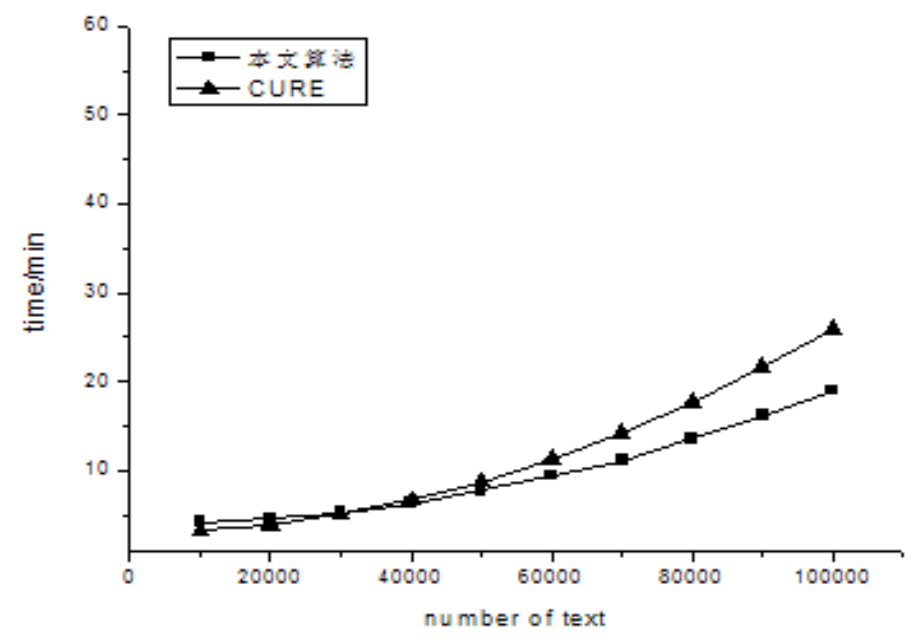

Figure 5. Time performance comparison.

Table 1. Clustering effect comparison between our algorithm and CURE algorithm

\begin{tabular}{cccccc}
\hline & Clusters & Big categories/texts & Small categories/texts & ACC & NMI \\
\hline Our algorithm & 2275 & $161 / 5764$ & $2114 / 4236$ & $74.3 \%$ & $69.1 \%$ \\
$\begin{array}{c}\text { CURE } \\
\text { algorithm }\end{array}$ & 2083 & $143 / 6108$ & $1940 / 3892$ & $72.1 \%$ & $63.1 \%$ \\
\hline
\end{tabular}


number of texts is greater than or equal to 5 is called big category. The experiment results are shown in Table $\mathbf{1 .}$ As can be seen from the data, numbers of big categories of two algorithms are both larger than the 133 categories marked artificially, and text algorithm is especially large. By observing the clustering results, we can find that some clusters are similar in the cluster sets obtained by text algorithm, but there are also some noise data in cluster sets, and cluster do not merge. So we speculate that if the result of hierarchical clustering is not good, there will be noise data in cluster sets, and then this algorithm will not merge them but make them separate clusters. That is to say, in part, we have discreteness among clusters exchange for compactness within cluster, which make noise convergence in iteration. So our algorithm is slightly higher than CURE in ACC and NMI. In addition, they both do well when dealing with isolated points and abnormal points.

Finally, we tested their performance in time from the 103048 short texts. Time change is shown in Figure 5.

From Figure 5, we can see that the two algorithms are of polynomial time complexity. In addition, our algorithm has a lower growth rate.

\section{Conclusion}

This paper studies volume short texts clustering problems produced by mobile Internet. We propose a two-stage clustering strategy using sliding windows according to characteristics of short texts of volume data and dynamic growth, whose time complexity is $\mathrm{O}\left(\mathrm{N}^{2}\right)$. We did hierarchical clustering in sliding window, and merged hierarchical clustering results using information gain theory. The experiment results show that this method has considered the global feature of clusters when clustering, so it is of high accuracy. It can be easily extended to multi-threaded and distributed algorithm, which has a good application prospect in large-scale short texts clustering.

\section{References}

[1] He, H., Chen, B., Xu, W., et al. (2007) Short Text Feature Extraction and Clustering for Web Topic Mining. IEEE Third International Conference on Semantics, Knowledge and Grid, 382-385.

[2] Hartigan, J.A. and Wong, M.A. (1979) Algorithm AS 136: A k-Means Clustering Algorithm. Journal of the Royal Statistical Society, Series C (Applied Statistics), 28, 100-108.

[3] Szekely, G.J. and Rizzo, M.L. (2005) Hierarchical Clustering via Joint between-within Distances: Extending Ward’s Minimum Variance Method. Journal of Classification, 22, 151-183. http://dx.doi.org/10.1007/s00357-005-0012-9

[4] Zhao, P. and Cai, Q.S. (2007) Research of Novel Chinese Text Clustering Algorithm Based on HowNet. Computer Engineering and Applications, 43, 162-163.

[5] Tang, J., Wang, X., Gao, H., et al. (2012) Enriching Short Text Representation in Microblog for Clustering. Frontiers of Computer Science, 6, 88-101.

[6] Wang, L., Jia, Y., Han, W. (2007) Instant Message Clustering Based on Extended Vector Space Model. Advances in Computation and Intelligence, Springer Berlin Heidelberg, 435-443. http://dx.doi.org/10.1007/978-3-540-74581-5_48

[7] Peng, Z.Y., Yu, X.M., Xu H.B., et al. (2011) Incomplete Clustering for Large Scale Short Texts. Journal of Chinese Information, 25, 54-59.

[8] Chen, J.C., Hu, G.W., Yang, Z.H., et al. (2011) Text Clustering Based on Global Center-Determination. Computer Engineering and Applications, 47, 147-150.

[9] Liu, Z.X., Liu, Y.B. and Luo, L.M. (2010) An Efficient Density and Grid Based Clustering Algorithm. Journal of Chongqing University of Posts and Telecommunications (Natural Science Edition), 22, 242-247.

[10] Quinlan, J.R. (1979) Discovering Rules by Induction from Large Collections of Examples. Expert Systems in the Micro Electronic Age. Edinburgh University Press.

[11] Guha, S., Rastogi, R. and Shim, K. (1998) CURE: An Efficient Clustering Algorithm for Large Databases. ACM SIGMOD Record, ACM, 27, 73-84.

[12] Zhou, Z.T. (2005) Quality Evaluation of Text Clustering Results and Investigation on Text Representation. Graduate University of Chinese Academy of Sciences, Beijing. 